\title{
Author Correction: Human mesenchymal stem cells promote tumor growth via MAPK pathway and metastasis by epithelial mesenchymal transition and integrin a5 in hepatocellular carcinoma
}

\author{
Jiang Chen ${ }^{1}$, Tong $\mathrm{Ji}^{1}$, Di Wu', Shi Jiang ${ }^{1}$, Jie Zhao ${ }^{1}$, Hui Lin ${ }^{1}$ and Xiujun Cai ${ }^{1}$
}

\section{Correction to: Cell Death and Disease https://doi.org/10.1038/s41419-019-1622-1 \\ published online 29 May 2019}

Since publication of this article, the authors noticed that the author list had been changed from "Jiang $\mathrm{Chen}^{1}$, Tong $\mathrm{Ji}^{1}$, Di Wu ${ }^{1}$, Shi Jiang ${ }^{1}$, Jie Zhao ${ }^{1}$, Hui Lin ${ }^{1}$ and Xiujun $\mathrm{Cai}^{1}$ " to "Jiang Chen ${ }^{1}$, Tong $\mathrm{Ji}^{1}$, Di Wu ${ }^{1}$, Shi Jiang ${ }^{1}$, Jie Zhao ${ }^{1}$, Xiujun Cai1 and Hui Lin1"
The correct list should be "Jiang Chen" ${ }^{1}$, Tong Ji 1, Di $\mathrm{Wu}^{1}$, Shi Jiang ${ }^{1}$, Jie Zhao ${ }^{1}$, Hui $\operatorname{Lin}^{1}$ and Xiujun $\mathrm{Cai}^{1}{ }^{1}$,

This has been corrected in both the PDF and HTML versions of the Article.

Published online: 20 June 2019 